\title{
GLOBAL WEAK SOLUTIONS TO THE NAVIER-STOKES EQUATIONS FOR A 1D VISCOUS POLYTROPIC IDEAL GAS
}

\author{
BY
}

SONG JIANG (LCP, Institute of Applied Physics and Computational Mathematics, P.O. Box 8009, Beijing 100088, China)

AND

PING ZHANG (Academy of Mathematics and System Sciences, Chinese Academy of Sciences, Beijing 100080, China)

\begin{abstract}
We prove the existence of global weak solutions to the Navier-Stokes equations for a one-dimensional viscous polytropic ideal gas. We require only that the initial density is in $L^{\infty} \cap L_{\text {loc }}^{2}$ with positive infimum, the initial velocity is in $L_{\text {loc }}^{2}$, and the initial temperature is in $L_{\text {loc }}^{1}$ with positive infimum. The initial density and the initial velocity may have differing constant states at $x= \pm \infty$. In particular, piecewise constant data with arbitrary large jump discontinuities are included. Our results show that neither vacuum states nor concentration states can form and the temperature remains positive in finite time.
\end{abstract}

1. Introduction. We study the global existence of weak solutions to the Cauchy problem for the Navier-Stokes equations for a one-dimensional viscous polytropic ideal gas:

$$
\begin{aligned}
& \rho_{t}+(\rho v)_{x}=0 \\
& (\rho v)_{t}+\left(\rho v^{2}\right)_{x}+P_{x}=\mu v_{x x} \\
& {\left[\rho\left(c_{V} \theta+\frac{v^{2}}{2}\right)\right]_{t}+\left[v\left(\rho \frac{v^{2}}{2}+c_{V} \rho \theta+P\right)\right]_{x}=\kappa \theta_{x x}+\mu\left(v v_{x}\right)_{x}}
\end{aligned}
$$

with initial conditions

$$
(\rho(x, 0), v(x, 0), \theta(x, 0))=\left(\rho_{0}(x), v_{0}(x), \theta_{0}(x)\right), x \in \mathbb{R} .
$$

Received October 3, 2000 and, in revised form, March 4, 2002.

2000 Mathematics Subject Classification. Primary 76N10, 35M10, 35Q30.

Key words and phrases. Compressible Navier-Stokes equations, global weak solutions.

E-mail address: jiang@mail.iapcm.ac.cn

E-mail address: zp@math03.math.ac.cn 
Here $\rho, v$, and $\theta$ denote the density, the velocity, and the absolute temperature, respectively, $P=P(\rho, \theta)$ is the pressure having the form (ideal gases)

$$
P=R \rho \theta
$$

$\mu, c_{V}, \kappa, R$ are positive constants, and at infinity, the initial data $\rho_{0}, v_{0}, \theta_{0}$ satisfy

$$
\lim _{x \rightarrow \pm \infty}\left(\rho_{0}(x), v_{0}(x), \theta_{0}(x)\right)=\left(\rho^{ \pm}, v^{ \pm}, \bar{\theta}\right),
$$

where $\rho^{ \pm}, \bar{\theta}$ are positive constants and $v^{ \pm}$are constants satisfying $v^{-} \leq v^{+}$.

To describe the assumptions on the initial data, we define smooth functions $0<\bar{\rho}(x)$, $\bar{v}(x) \in C^{\infty}(\mathbb{R})$ satisfying

$$
\bar{\rho}(x)=\left\{\begin{array}{ll}
\rho^{+}, & x \geq 1, \\
\rho^{-}, & x \leq-1,
\end{array} \quad \bar{v}(x)= \begin{cases}v^{+}, & x \geq 1 \\
v^{-}, & x \leq-1 .\end{cases}\right.
$$

Then our hypotheses upon the initial data are that

$$
\left\{\begin{array}{l}
\rho_{0} \in L^{\infty}(\mathbb{R}), \inf _{\mathbb{R}} \rho_{0}(\cdot)>0, \rho_{0}-\bar{\rho}, v_{0}-\bar{v} \in L^{2}(\mathbb{R}), \\
\frac{\theta_{0}}{\bar{\theta}}-\ln \frac{\theta_{0}}{\bar{\theta}}-1 \in L^{1}(\mathbb{R}), \inf _{\mathbb{R}} \theta_{0}(\cdot)>0 .
\end{array}\right.
$$

Since the first work of Kazhikhov and Shelukhin [14] on the global existence in the dynamics of a one-dimensional compressible viscous heat-conducting fluid for large initial data, significant progress has been made on the mathematical aspect of the initial and initial boundary value problems for (1.1)-(1.3). For initial data in $H^{1}$, the existence and uniqueness of global generalized (strong) solutions to the Cauchy problem and to different initial boundary value problems have been known. Moreover, the global solution is asymptotically stable as time tends to infinity; see, for example, $[13,1,16,17,18,6$, 10, 11] and others, where $\rho^{+}=\rho^{-}$and $v^{ \pm}=0$ are assumed. In [9] global generalized solutions to the Cauchy problem were studied for the initial data in $H^{1}$ where differing end states $\rho^{ \pm}, v^{ \pm}$with $v^{-} \leq v^{+}$are allowed.

Concerning weak solutions, Serre in 1986 [20] proved the global existence of weak solutions to the Cauchy problem under the conditions that $\rho_{0} \in B V, \rho^{+}=\rho^{-}$, and $v^{ \pm}=0$. For the initial data satisfying $\left(\rho_{0}, v_{0}, \theta_{0}\right) \in L^{\infty} \times L^{2} \times L^{1}$, inf $\rho_{0}>0$, and $\theta_{0}>0$ a.e., Amosov and Zlotnik [2, 3, 21] obtained global weak solutions of initial boundary value problems in bounded intervals (see also [6] for nonnegative initial density and $[12,7]$ for small initial data). Recently, Hoff [8] studied the Navier-Stokes equations of compressible isentropic flow and proved the existence of global weak solutions for $\rho_{0} \in L^{\infty}$ with positive infimum, $v_{0} \in L^{2}$ with differing end states. And Chen, Hoff, and Trivisa [4] proved the global existence and large-time behaviour of weak solutions to (1.1-1.3) in bounded domain. To our best knowledge, the problem concerning the global existence of weak solutions to the Cauchy problem (1.1)-(1.4) under (1.6) and (1.8) still remains open.

Our aim in this paper is to prove a global existence theorem on weak solutions to (1.1)-(1.4). In particular, our result allows piecewise constant data with arbitrary large jump discontinuities. An important physical consequence of our result is that neither vacuum states $(\rho=0)$ nor concentration states $(\rho=\infty)$ can occur in the solution and the temperature remains positive, no matter how large the oscillation of initial data. 
Before stating the main result of the present paper we introduce the notation used throughout this paper: Let $s$ be a real number and let $1 \leq p \leq \infty$. By $W^{s, p}$ we denote the usual Sobolev space defined over $\mathbb{R}$ with norm $\|\cdot\|_{W^{s, p}} ; H^{s} \equiv W^{s, 2}$ with norm $\|\cdot\|_{H^{s}}$, $L^{p} \equiv W^{0, p}$ with norm $\|\cdot\|_{L^{p}} . W_{\mathrm{loc}}^{s, p}, H_{\mathrm{loc}}^{s}$, and $L_{\mathrm{loc}}^{p}$ denote $W_{\mathrm{loc}}^{s, p}(\mathbb{R}), H_{\mathrm{loc}}^{s}(\mathbb{R})$, and $L_{\mathrm{loc}}^{p}(\mathbb{R})$, respectively. $L^{p}(I, B)$ resp. $\|\cdot\|_{L^{p}(I, B)}$ denotes the space of all strongly measurable, $p$ thpower integrable (essentially bounded if $p=\infty$ ) functions from $I$ to $B$ resp. its norm, $I \subset \mathbb{R}$ an interval, $B$ a Banach space. We also use $\mathbb{R}_{+}:=[0, \infty)$. The same letter $C$ (sometimes used as $C(X, \ldots)$ to emphasize the dependence of $C$ on $X, \ldots$ ) will denote various positive constants which may depend on $T$ but not on $\epsilon$.

Now we introduce the definition of weak solutions.

DEFInition. We say that $(\rho(x, t), v(x, t), \theta(x, t))$ is a global weak solution of (1.1)(1.4), if, for any $T>0, \rho(x, t) \geq 0$ and $\theta(x, t)>0$ on $[0, T] \times \mathbb{R}$, and

$$
\begin{gathered}
\rho \in L_{\mathrm{loc}}^{\infty}\left(\mathbb{R}_{+}, L^{\infty}\right), \rho-\bar{\rho}, v-\bar{v} \in L_{\mathrm{loc}}^{\infty}\left(\mathbb{R}_{+}, L^{2}\right), v \in L_{\mathrm{loc}}^{2}\left(\mathbb{R}_{+}, H_{\mathrm{loc}}^{1}\right), \\
\frac{\theta}{\bar{\theta}}-\log \frac{\theta}{\bar{\theta}}-1 \in L_{\mathrm{loc}}^{\infty}\left(\mathbb{R}_{+}, L^{1}\right), \theta \in L_{\mathrm{loc}}^{\infty}\left(\mathbb{R}_{+}, L_{\mathrm{loc}}^{1}\right) \cap L_{\mathrm{loc}}^{2}\left(\mathbb{R}_{+} \times \mathbb{R}\right) \cap L_{\mathrm{loc}}^{1}\left(\mathbb{R}_{+}, W_{\mathrm{loc}}^{1,1}\right),
\end{gathered}
$$

and the following equations hold:

$$
\begin{aligned}
& \int_{\mathbb{R}_{+}} \int_{\mathbb{R}}\left\{\rho \phi_{t}+\rho v \phi_{x}\right\} d x d t=\int_{\mathbb{R}} \rho_{0} \phi(x, 0) d x \\
& \int_{\mathbb{R}_{+}} \int_{\mathbb{R}}\left\{\rho v \phi_{t}+\rho v^{2} \phi_{x}+P \phi_{x}-\mu v_{x} \phi_{x}\right\} d x d t=\int_{\mathbb{R}} \rho_{0} v_{0} \phi(x, 0) d x \\
& \int_{\mathbb{R}_{+}} \int_{\mathbb{R}}\left\{\rho\left(c_{V} \theta+\frac{v^{2}}{2}\right) \phi_{t}+v\left(\rho \frac{v^{2}}{2}+c_{V} \rho \theta+P\right) \phi_{x}-\kappa \theta_{x} \phi_{x}+\mu \frac{v^{2}}{2} \phi_{x x}\right\} d x d t \\
& \quad=\int_{\mathbb{R}} \rho_{0}\left(c_{V} \theta_{0}+\frac{v_{0}}{2}\right) \phi(x, 0) d x
\end{aligned}
$$

for any $\phi \in C_{0}^{\infty}\left(\mathbb{R}_{+} \times \mathbb{R}\right)$.

Thus, the main result of this paper reads:

TheOREM 1.1. Assume that $\rho_{0}, v_{0}, \theta_{0}$ satisfy (1.8). Then there exists a global weak solution $(\rho, v, \theta)$ of (1.1)-(1.4), such that for all $p<3 / 2, \theta_{x} \in L_{\text {loc }}^{p}\left(\mathbb{R}_{+} \times \mathbb{R}\right)$, and for any $T>0, v_{x} \in L_{\text {loc }}^{2}\left(\mathbb{R}_{+}, L^{2}(\mathbb{R})\right)$,

$$
\begin{gathered}
C_{1} \leq \rho(x, t) \leq C_{2}, C_{1} \leq \theta(x, t), \quad \text { for a.e. } x \in \mathbb{R}, t \in[0, T], \\
\int_{\mathbb{R}}\left\{\left(\frac{\bar{\rho}}{\rho}-\log \frac{\bar{\rho}}{\rho}-1\right)+\left(\frac{\theta}{\bar{\theta}}-\log \frac{\theta}{\bar{\theta}}-1\right)+(v-\bar{v})^{2}\right\}(x, t) d x \\
\leq C \quad \forall t \in[0, T],
\end{gathered}
$$

where $C_{1}, C_{2}, C$ are positive constants which may depend on $T$.

The proof of Theorem 1.1 is based on passing to the limit for the smooth approximate solutions and a basic energy estimate which is deduced by using some relations associated with the second law of thermodynamics. Due to the differing end states, some difficulties arise in the derivation of the energy estimate. We can overcome these difficulties by exploiting the facts that $v^{-} \leq v^{+}$and that the end states for $\theta_{0}$ are the same. The boundedness of $\rho$ from below and above can be obtained by using arguments similar 
to those in $[13,1]$ while lower bounds of $\theta$ can be achieved by applying the maximum principle for linear parabolic equations in unbounded domains. In order to pass to the limit for the smooth approximate solutions we apply Lemma 5.1 of Lions [15, p. 12] on weak compactness. This needs the estimates of some derivatives of $(\rho, v, \theta)$ in some $L^{p}$ spaces, and we use the basic energy estimate and the interpolation theory to derive the estimates of the derivatives. In Sec. 2 we derive uniform a priori estimates for the approximate solutions and the proof of Theorem 1.1 is given in Sec. 3 .

2. Uniform a priori estimates for the approximate solutions. In this section we derive some uniform a priori estimates for the approximate solutions which are obtained by mollifying the initial data.

Let $j_{\epsilon}(x)$ denote the standard Friedrichs' mollifier, let $\rho_{0}^{\epsilon}(x):=\rho_{0} * j_{\epsilon}, v_{0}^{\epsilon}(x):=v_{0} * j_{\epsilon}$, $\theta_{0}^{\epsilon}(x):=\theta_{0} * j_{\epsilon}$. Then under the conditions of Theorem 1.1, let us consider the Cauchy problem $(1.1)-(1.4)$ with $\left(\rho_{0}, v_{0}, \theta_{0}\right)$ replaced by $\left(\rho_{0}^{\epsilon}, v_{0}^{\epsilon}, \theta_{0}^{\epsilon}\right)$.

Firstly applying Jensen's inequality and (1.8), and taking into account $C_{1} \leq \theta_{0}^{\epsilon}(x) \leq$ $C(\epsilon)$ that follows from $\lim _{|x| \rightarrow \infty} \theta^{0}(x)=\bar{\theta}$ and the properties of mollifier, we have

$$
\begin{aligned}
& \int_{\mathbb{R}}\left(\frac{\theta_{0}^{\epsilon}}{\bar{\theta}}-\log \frac{\theta_{0}^{\epsilon}}{\bar{\theta}}-1\right) d x \leq \int_{\mathbb{R}}\left(\frac{\theta^{0}}{\bar{\theta}}-\ln \frac{\theta^{0}}{\bar{\theta}}-1\right) * j_{\epsilon} d x \leq \int_{\mathbb{R}}\left(\frac{\theta^{0}}{\bar{\theta}}-\ln \frac{\theta^{0}}{\bar{\theta}}-1\right) d x, \\
& \int_{\mathbb{R}}\left(\theta_{0}^{\epsilon}-\bar{\theta}\right)^{2} d x \leq C(\epsilon) \int_{\mathbb{R}}\left|\theta_{0}^{\epsilon}-\bar{\theta}\right| d x \leq C(\epsilon) .
\end{aligned}
$$

Noting that $|x-\log x-1| \leq C|x-1|^{2}$ for $\delta \leq x \leq C_{1}$, we get

$$
\begin{aligned}
\int_{\mathbb{R}}\left(\frac{\bar{\rho}}{\rho_{0}^{\epsilon}}-\log \frac{\bar{\rho}}{\rho_{0}^{\epsilon}}-1\right) d x & \leq C \int_{\mathbb{R}}\left(\rho_{0}^{\epsilon}-\bar{\rho}\right)^{2} d x \\
& \leq C \int_{\mathbb{R}}\left\{\left(\rho_{0}-\bar{\rho}\right)^{2}+\left(\bar{\rho} * j_{\epsilon}-\bar{\rho}\right)^{2}\right\} d x \leq C
\end{aligned}
$$

For the sake of simplicity we will drop the subscript $\epsilon$ throughout this section.

To construct the approximate solutions $(\rho(x, t), v(x, t), \theta(x, t))$ and to derive the a priori estimates, it is convenient to use Lagrangian coordinates instead of Eulerian coordinates $(x, t)$. We introduce the coordinate transformation:

$$
y=\int_{0}^{x} \rho(\xi, t) d \xi-\int_{0}^{t}(\rho v)(0, \tau) d \tau \equiv r(x, t), \quad \tau=t
$$

We will prove later that $\rho(x, t)$ is bounded and strictly away from 0 , then for each $t \geq 0$ the mapping (the inverse mapping of $r(x, t)) x \equiv x(y, t): \mathbb{R} \rightarrow \mathbb{R}$ is surjective. Hence, we can reformulate the problem (1.1)-(1.4) in Lagrangian coordinates $(y, \tau)$; that is, if we denote $u:=1 / \rho$, the specific volume, and use $t$ instead of $\tau$ in the case without confusion, 
then the problem (1.1)-(1.4) with (1.6) is transformed into

$$
\begin{aligned}
u_{t} & =v_{y}, \\
v_{t} & =\sigma_{y}, \quad\left(\sigma:=\mu \frac{v_{y}}{u}-R \frac{\theta}{u}\right) \\
c_{V} \theta_{t} & =\sigma v_{y}+\kappa\left[\frac{\theta_{y}}{u}\right]_{y}
\end{aligned}
$$

with the initial data

$$
(u(y, 0), v(y, 0), \theta(y, 0))=\left(u_{0}(y), v_{0}(y), \theta_{0}(y)\right), \quad y \in \mathbb{R}
$$

and the conditions at infinity

$$
\lim _{y \rightarrow \pm \infty}(u(y, t), v(y, t), \theta(y, t))=\left(u^{ \pm}, v^{ \pm}, \bar{\theta}\right) \text { with } u^{ \pm}:=1 / \rho^{ \pm} .
$$

Now we take functions $\bar{u}(y), \bar{v}(y) \in C^{\infty}(\mathbb{R})$ with $\bar{u}>0, \bar{v}_{y} \geq 0$ and

$$
\bar{u}(y)=\left\{\begin{array}{ll}
u^{+}, & y \geq 1, \\
u^{-}, & y \leq-1,
\end{array} \quad \bar{v}(y)= \begin{cases}v^{+}, & y \geq 1 \\
v^{-}, & y \leq-1\end{cases}\right.
$$

Then, by (1.8), similar conditions to (1.8) still hold for $\left(u_{0}(y), v_{0}(y), \theta_{0}(y)\right)$. It should be pointed out here that because of $u^{ \pm}>0$ and $v^{-} \leq v^{+}$we can easily construct functions $\bar{u}$ and $\bar{v}$ which satisfy $\bar{u}>0, \bar{v}_{y} \geq 0$.

Thus by the following Lemma 2.1, Lemma 2.2, and modifying the method of [9], we can prove that (2.4)-(2.8) possesses a unique global strong solution $(u, v, \theta)$; moreover, $u$ is bounded and strictly away from 0 . Thus by (2.3), we get a unique global strong solution $(\rho(x, t), v(x, t), \theta(x, t))$ for the Cauchy problem $(1.1)-(1.4)$ with initial data $\left(\rho_{0}^{\epsilon}, v_{0}^{\epsilon}, \theta_{0}^{\epsilon}\right)$.

We have the following basic energy estimate which is obtained by using some relations associated with the second law of thermodynamics and which embodies the dissipation induced by viscosity and thermal diffusion.

LEMMA 2.1. Let

$$
W(y, t):=R \bar{\theta}\left(\frac{u}{\bar{u}}-\log \frac{u}{\bar{u}}-1\right)+c_{V} \bar{\theta}\left(\frac{\theta}{\bar{\theta}}-\log \frac{\theta}{\bar{\theta}}-1\right) .
$$

Then we have

$$
\int_{\mathbb{R}}\left[W(y, t)+\frac{1}{2}(v(y, t)-\bar{v}(y))^{2}\right] d y+\bar{\theta} \int_{0}^{t} \int_{\mathbb{R}}\left[\mu \frac{v_{y}^{2}}{u \theta}+\kappa \frac{\theta_{y}^{2}}{u \theta^{2}}\right] d y d s \leq C
$$

for all $t \in[0, T]$.

Proof. Using Eqs. (2.4)-(2.6), we obtain, after a straightforward calculation, that

$$
\begin{aligned}
{[W} & \left.+\frac{(v-\bar{v})^{2}}{2}\right]_{t}+\bar{\theta}\left[\mu \frac{v_{y}^{2}}{u \theta}+\kappa \frac{\theta_{y}^{2}}{u \theta^{2}}\right] \\
& =R \frac{\bar{\theta}}{\bar{u}} v_{y}+[\sigma(v-\bar{v})]_{y}+\sigma \bar{v}_{y}+\kappa\left[\frac{\theta y}{u}\left(1-\frac{\bar{\theta}}{\theta}\right)\right]_{y} .
\end{aligned}
$$


Recalling the definition of $\bar{u}$ and $\bar{v}$, we integrate the above equality over $\mathbb{R} \times(0, t)$ to infer that

$$
\begin{aligned}
\int_{\mathbb{R}}( & \left.W+\frac{(v-\bar{v})^{2}}{2}\right)(y, t) d y+\bar{\theta} \int_{0}^{t} \int_{\mathbb{R}}\left(\mu \frac{v_{y}^{2}}{u \theta}+\kappa \frac{\theta_{y}^{2}}{u \theta^{2}}\right) d y d s \\
& \leq \int_{\mathbb{R}}\left\{W(y, 0)+\left(\frac{v_{0}-\bar{v}}{2}\right)^{2}\right\} d y+\int_{0}^{t} \int_{\mathbb{R}}\left(R \frac{\bar{\theta}}{\bar{u}} v_{y}+\sigma \bar{v}_{y}\right) d y d s \\
& =E_{0}+\int_{0}^{t} \int_{\mathbb{R}}\left\{R \frac{\bar{\theta}}{\bar{u}} \bar{v}_{y}+R \frac{\bar{\theta}}{\bar{u}}(v-\bar{v})_{y}+\sigma \bar{v}_{y}\right\} d y d s \\
& \leq E_{0}+C-R \int_{0}^{t} \int_{\mathbb{R}}\left[\frac{\bar{\theta}}{\bar{u}}\right]_{y}(v-\bar{v}) d y d s+\int_{0}^{t} \int_{\mathbb{R}} \sigma \bar{v}_{y} d y d s,
\end{aligned}
$$

where (1.8), (2.1), (2.2), and the coordinate transformation (2.3) yield

$$
\begin{aligned}
E_{0} & :=\int_{\mathbb{R}}\left\{W(y, 0)+\frac{\left(v_{0}-\bar{v}\right)^{2}}{2}\right\}(y) d y \\
& \leq C \int_{\mathbb{R}}\left\{\left(u_{0}-\bar{u}\right)^{2}+\left|\theta_{0}-\bar{\theta}_{0}\right|+\left(v_{0}-\bar{v}\right)^{2}\right\}(y) d y+1 \\
& \leq C \int_{\mathbb{R}}\left\{\left(\rho_{0}-\bar{\rho}\right)^{2}+\left|\theta_{0}-\bar{\theta}\right|+\left(v_{0}-\bar{v}\right)^{2}\right\}(x) d x+1 \leq C .
\end{aligned}
$$

Observing that $\bar{v}_{y} \geq 0, \theta / u>0$, and $\operatorname{supp} \bar{v}_{y} \subset\{y|| y \mid \leq 1\}$, we obtain by Eq. (2.4) that

$$
\begin{aligned}
\int_{0}^{t} \int_{\mathbb{R}} \sigma \bar{v}_{y} d y d s & =-R \int_{0}^{t} \int_{\mathbb{R}} \frac{\theta}{u} \bar{v}_{y} d y d s+\mu \int_{0}^{t} \int_{\mathbb{R}} \frac{v_{y}}{u} \bar{v}_{y} d y d s \\
& \leq \mu \int_{0}^{t} \int_{\mathbb{R}}(\log u)_{t} \bar{v}_{y} d y d s \\
& =\mu \int_{\mathbb{R}} \bar{v}_{y} \log \frac{u}{\bar{u}} d y-\mu \int_{\mathbb{R}} \bar{v}_{y} \log \frac{u_{0}}{\bar{u}} d y \\
& =\mu \int_{\{y \mid u(y, t) \leq \bar{u}(y)\}} \bar{v}_{y} \log \frac{u}{\bar{u}} d y+\mu \int_{\{y \mid u(y, t)>\bar{u}(y)\}} \bar{v}_{y} \log \frac{u}{\bar{u}} d y+C \\
& \leq C+\mu \int_{\{y \mid u(y, t)>\bar{u}(y)\}} \bar{v}_{y} \log \frac{u}{\bar{u}} d y \\
& \leq C+C \int_{\{y|u(y, t)>\bar{u}(y),| y \mid \leq 1\}} \log \frac{u}{\bar{u}} d y .
\end{aligned}
$$

Note that for $x \geq 1, \log x \leq \sqrt{2}(x-\log x-1)^{1 / 2}$, we get from the above inequality that

$$
\begin{aligned}
\int_{0}^{t} \int_{\mathbb{R}} \sigma \bar{v}_{y} d y d s & \leq C \int_{\{y|u(y, t) \geq \bar{u}(y),| y \mid \leq 1\}}\left(\frac{u}{\bar{u}}-\log \frac{u}{\bar{u}}-1\right)^{1 / 2} d y+C \\
& \leq \varepsilon \int_{\mathbb{R}}\left(\frac{u}{\bar{u}}-\log \frac{u}{\bar{u}}-1\right) d y+C \varepsilon^{-1} \quad(0 \leq \varepsilon<1) .
\end{aligned}
$$


Substituting (2.12) into (2.11), taking $\varepsilon$ appropriately small, taking into account supp $\bar{u}_{y} \subset$ $\{y|| y \mid \leq 1\}$, and applying Cauchy-Schwarz's inequality, we conclude that

$$
\int_{\mathbb{R}}\left(W+(v-\bar{v})^{2}\right)(y, t) d y+\int_{0}^{t} \int_{\mathbb{R}}\left(\frac{v_{y}^{2}}{u \theta}+\frac{\theta_{y}^{2}}{u \theta^{2}}\right) d y d s \leq C+C \int_{0}^{t} \int_{\mathbb{R}}(v-\bar{v})^{2} d y d s,
$$

which, by applying Gronwall's inequality, immediately gives the lemma. The proof is complete.

With the above basic energy estimate, we are able to bound the specific volume $u(y, t)$ and the temperature $\theta(y, t)$.

Lemma 2.2. There are positive constants $C_{1}, C_{2}$ which may depend on $T$, such that

$$
C_{1} \leq u(y, t) \leq C_{2}, C_{1} \leq \theta(x, t) \quad \forall x \in \mathbb{R}, t \in[0, T] .
$$

Proof. The proof of the boundedness of $\rho$ is parallel to that for generalized (strong) solutions (see, e.g., $[1,13])$. For the sake of completeness we present the proof here.

It is easy to see that Lemma 2.1 implies

$$
\int_{i}^{i+1}\left(\frac{u}{\bar{u}}-\log \frac{u}{\bar{u}}-1\right)(y, t) d y, \int_{i}^{i+1}\left(\frac{\theta}{\bar{\theta}}-\log \frac{\theta}{\bar{\theta}}-1\right)(y, t) d y \leq C, \quad t \in[0, T] .
$$

Since $x-\log x-1$ is convex, one sees that

$$
\int_{i}^{i+1} \frac{u}{\bar{u}} d y-\log \int_{i}^{i+1} \frac{u}{\bar{u}} d y-1, \int_{i}^{i+1} \frac{\theta}{\bar{\theta}} d y-\log \int_{i}^{i+1} \frac{\theta}{\bar{\theta}} d y-1 \leq C, \quad t \in[0, T] .
$$

The estimates (2.14), (2.15) show that

$$
\alpha_{1} \leq \int_{i}^{i+1} u(y, t) d y, \int_{i}^{i+1} \theta(y, t) d y \leq \beta_{1}, \quad i=0, \pm 1, \pm 2, \ldots, t \in[0, T],
$$

and that by virtue of the mean value theorem, for each $t \in[0, T]$ there are $a_{i}(t), b_{i}(t) \in$ $[i, i+1]$, such that

$$
\alpha_{1} \leq u\left(a_{i}(t), t\right), \theta\left(b_{i}(t), t\right) \leq \beta_{1}, \quad i=0, \pm 1, \pm 2, \ldots,
$$

where $\alpha_{1}, \beta_{1}$ are the positive roots of the equation $x-\log x-1=C$.

We may write Eq. (2.5) as $v_{t}=-R[\theta / u]_{y}+\mu[\log u]_{t y}$. Integrating this equation first over $(0, t)$ with respect to $t$, then over $\left(a_{i}(t), y\right)(y \in[i, i+1])$ with respect to $y$, and taking exponential on both sides of the resulting equation, we obtain

$$
\frac{1}{u(y, t)} \exp \left\{\frac{R}{\mu} \int_{0}^{t} \frac{\theta(y, s)}{u(y, s)} d s\right\}=B_{i}(y, t) Y_{i}(t), \quad y \in[i, i+1]
$$

where

$$
\begin{aligned}
B_{i}(y, t) & :=\frac{u_{0}\left(a_{i}(t)\right)}{u\left(a_{i}(t), t\right) u_{0}(y)} \exp \left\{-\frac{1}{\mu} \int_{a_{i}(t)}^{y}\left(v-v_{0}\right) d \xi d s\right\}, \\
Y_{i}(t) & :=\exp \left\{\frac{R}{\mu} \int_{0}^{t} \frac{\theta\left(a_{i}(t), s\right)}{u\left(a_{i}(t), s\right)} d s\right\} .
\end{aligned}
$$

Multiplying (2.18) by $R \theta / \mu$ and integrating over $[0, t]$, we arrive at

$$
\exp \left\{\int_{0}^{t} \frac{\theta(y, s)}{u(y, s)} d s\right\}=1+\frac{R}{\mu} \int_{0}^{t} B_{i}(y, s) Y_{i}(s) \theta(y, s) d s .
$$


Inserting the above identity into (2.18), we immediately get the following representation for $u(y, t)$ :

$$
u(y, t)=\frac{1}{B_{i}(y, t) Y_{i}(t)}\left\{1+\frac{R}{\mu} \int_{0}^{t} B_{i}(y, s) Y_{i}(s) \theta(y, s) d s\right\}
$$

for $y \in[i, i+1], t \geq 0$ and $i=0, \pm 1, \pm 2, \ldots$ Recalling the definition of $B_{i}$ and $Y_{i}$, using Cauchy-Schwarz's inequality, Lemma 2.1, and (2.17), we see that

$$
0<C^{-1} \leq B_{i}(y, t) \leq C, Y_{i}(t) \geq 1, y \in[i, i+1], t \in[0, T], \quad i=0, \pm 1, \pm 2, \ldots .
$$

Now we integrate $(2.19)$ over $(i, i+1)$ with respect to $y$ and make use of $(2.16)$ and $(2.20)$ to obtain $Y_{i}(t) \leq C+C \int_{0}^{t} Y_{i}(s) d s$, which together with Gronwall's inequality gives

$$
Y_{i}(t) \leq C \text { for all } t \in[0, T], \quad i=0, \pm 1, \pm 2, \ldots .
$$

It follows from (2.17) that

$$
\begin{aligned}
\theta(y, t) & \leq C\left(\theta^{1 / 2}(y, t)-\theta^{1 / 2}\left(b_{i}(t), t\right)\right)^{2}+C \\
& \leq C+C\left(\int_{i}^{i+1} \frac{\left|\theta_{y}\right|}{\theta^{1 / 2}} d y\right)^{2} \\
& \leq C+C \int_{i}^{i+1} \frac{\theta_{y}^{2}}{u \theta^{2}} d y \max _{[i, i+1]} u(\cdot, t), \quad y \in[i, i+1] .
\end{aligned}
$$

Inserting (2.20)-(2.22) into (2.19), we find that

$$
u(y, t) \leq C+C \int_{0}^{t} \int_{\mathbb{R}} \frac{\theta_{y}^{2}}{u \theta^{2}} d y \max _{[i, i+1]} u(\cdot, s) d s \quad \forall y \in[i, i+1], t \in[0, T] .
$$

From this, Gronwall's inequality, and Lemma 2.1, we obtain $u(y, t) \leq C_{2}$ for all $y \in$ $[i, i+1], t \in[0, T]$ and $i=0, \pm 1, \pm 2, \ldots$ On the other hand, from (2.19)-(2.21), one easily gets $u(y, t) \geq C_{1}$ for all $y \in[i, i+1], t \in[0, T]$ and $i=0, \pm 1, \pm 2, \ldots$ Thus, we have proved the lower and upper boundedness of $\rho$.

To show that the temperature is bounded below away from zero, we apply the maximum principle. To this end we write Eq. (2.6), using the estimate $C_{1} \leq u(y, t) \leq C_{2}$, as follows:

$$
\begin{aligned}
c_{V}\left[\frac{1}{\theta}\right]_{t} & =-\mu \frac{v_{y}^{2}}{u \theta^{2}}+R \frac{v_{y}}{u \theta}+\kappa\left[\frac{1}{u}\left(\frac{1}{\theta}\right)_{y}\right]_{y}-2 \kappa \frac{\theta_{y}^{2}}{u \theta^{3}} \\
& \leq C+\kappa\left[\frac{1}{u}\left(\frac{1}{\theta}\right)_{y}\right]_{y}, \quad y \in \mathbb{R}, t \in[0, T]
\end{aligned}
$$

where the positive constant $C$ is independent of $\epsilon$. Applying the maximum principle for parabolic equations in unbounded domains [19, Chapter 3], we obtain $1 / \theta(y, t) \leq C$ for all $x \in \mathbb{R}$ and $t \in[0, T]$, which gives the lower boundedness of $\theta$. This completes the proof of the lemma.

Based on the results established in Lemmas 2.1-2.2, we show the following estimates which will be important in the limit procedure in the next section. 
Lemma 2.3. Let $M \in \mathbb{N}$. Then we have

$$
\begin{aligned}
& \int_{0}^{T} \sup _{\mathbb{R}} \theta(t, \cdot) d t \leq C, \int_{0}^{T} \int_{\mathbb{R}} v_{y}^{2} d y d t \leq C_{T}, \\
& \int_{0}^{T} \sup _{[-M, M]} v^{2}(\cdot, t) d t+\int_{0}^{T} \int_{-M}^{M} \theta^{2} d y d t \leq C(M), \\
& \int_{0}^{T} \int_{-M}^{M}\left|\theta_{y}\right|^{p} d y d t \leq C(M, p) \quad \text { for all } p<3 / 2 .
\end{aligned}
$$

Proof. By virtue of (2.16)-(2.17), (2.13), and Lemma 2.1, one finds that

$$
\begin{aligned}
\int_{0}^{T} \max _{[i, i+1]} \theta(\cdot, t) d t & \leq C+\int_{0}^{T} \max _{[i, i+1]}\left|\theta(\cdot, t)-\theta\left(b_{i}(t), t\right)\right| d t \\
& \leq C+\int_{0}^{T} \int_{i}^{i+1}\left|\theta_{y}\right| d y d t \\
& \leq C+\left(\int_{0}^{T} \int_{i}^{i+1} \frac{\theta_{y}^{2}}{\theta^{2}} d y d t\right)^{1 / 2}\left(\int_{0}^{T} \int_{i}^{i+1} \theta^{2} d y d t\right)^{1 / 2} \\
& \leq C\left\{1+\left(\int_{0}^{T} \max _{[i, i+1]} \theta(\cdot, t) d t\right)^{1 / 2}\right\}, \quad i=0, \pm 1, \pm 2, \ldots,
\end{aligned}
$$

which implies $(2.24)_{1}$. Moreover, from $(2.16)$ and $(2.24)_{1}$, one gets

$$
\int_{0}^{T} \int_{-M}^{M} \theta^{2} d y d t \leq \int_{0}^{T} \sup \theta(\cdot, t) \int_{-M}^{M} \theta d y d t \leq C(M),
$$

which yields

$$
\begin{aligned}
\int_{0}^{T} \int_{i}^{i+1}\left|\theta_{y}\right| d y d t & \leq\left(\int_{0}^{T} \int_{i}^{i+1} \frac{\theta_{y}^{2}}{\theta^{2}} d y d t\right)^{1 / 2}\left(\int_{0}^{T} \int_{i}^{i+1} \theta^{2} d y d t\right)^{1 / 2} \\
& \leq C, \quad i=0, \pm 1, \pm 2, \ldots
\end{aligned}
$$

On the other hand, motivated by [9], we multiply $(2.5)$ by $(v-\bar{v})$ and integrate over $\mathbb{R}$ to get

$$
\begin{gathered}
\frac{1}{2} \frac{d}{d t} \int_{\mathbb{R}}(v-\bar{v})^{2} d y+\mu \int_{\mathbb{R}} \frac{v_{y}^{2}}{u} d y+R \int_{\mathbb{R}} \frac{\theta}{u} \bar{v}_{y} d y \\
=\mu \int_{\mathbb{R}} \frac{v_{y}}{u} \bar{v}_{y} d y+R \int_{\mathbb{R}} \frac{\theta}{u} v_{y} d y .
\end{gathered}
$$

Next we split up the second integral of (2.27) as follows:

$$
\int_{\mathbb{R}} \frac{\theta}{u} v_{y} d y=\int_{\mathbb{R}} \frac{\theta-\bar{\theta}}{u} v_{y} d y+\int_{\mathbb{R}} \frac{\bar{\theta}}{u} v_{y} d y=I_{1}+I_{2} .
$$

For $I_{1}$, we consider the following domains:

$$
\begin{aligned}
& \Omega_{1}(t)=\left\{x \mid \theta(t, y)>C_{0} \bar{\theta}\right\}, \quad C_{0}>>1, \\
& \Omega_{2}(t)=\left\{x \mid \theta(t, y) \leq C_{0} \bar{\theta}\right\} .
\end{aligned}
$$


Then one trivially has

$$
\left|\frac{\frac{\theta}{\bar{\theta}}-1}{\sqrt{\left(\frac{\theta}{\bar{\theta}}-\log \frac{\theta}{\bar{\theta}}-1\right)}}\right| \leq \sqrt{\frac{\theta}{\bar{\theta}}}, \quad x \in \Omega_{1}(t),
$$

and by the second inequality of (2.13), we have

$$
\left|\frac{\frac{\theta}{\bar{\theta}}-1}{\sqrt{\left(\frac{\theta}{\bar{\theta}}-\log \frac{\theta}{\bar{\theta}}-1\right)}}\right| \leq C, \quad x \in \Omega_{2}(t) \text {. }
$$

Thus by combining $(2.10),(2.13),(2.29)$ with $(2.30)$, we get

$$
\begin{aligned}
I_{1}= & \int_{\mathbb{R}} \frac{\bar{\theta} v_{y}}{u} \sqrt{\left(\frac{\theta}{\bar{\theta}}-\log \frac{\theta}{\bar{\theta}}-1\right)} \cdot\left|\frac{\frac{\theta}{\bar{\theta}}-1}{\sqrt{\left(\frac{\theta}{\bar{\theta}}-\log \frac{\theta}{\bar{\theta}}-1\right)}}\right| d x \\
\leq & \int_{\Omega_{1}(t)} \sqrt{\theta}\left|\frac{v_{y}}{u}\right| \sqrt{\bar{\theta}} \sqrt{\left(\frac{\theta}{\bar{\theta}}-\log \frac{\theta}{\bar{\theta}}-1\right)} d x \\
& +C \int_{\Omega_{2}(t)} \bar{\theta}\left|\frac{v_{y}}{u}\right| \sqrt{\left(\frac{\theta}{\bar{\theta}}-\log \frac{\theta}{\bar{\theta}}-1\right)} d x \\
\leq & \delta \int_{\mathbb{R}} \frac{v_{y}^{2}}{u} d y+C(\delta) \max _{x} \theta+C .
\end{aligned}
$$

While

$$
\begin{aligned}
I_{2} & =\frac{d}{d t} \int_{\mathbb{R}} \bar{\theta} \ln u d x=\frac{d}{d t} \int_{\mathbb{R}} \bar{\theta} \ln \frac{u}{\bar{u}} d x \\
& =-\frac{d}{d t} \int_{\mathbb{R}} \bar{\theta}\left(\frac{u}{\bar{u}}-\ln \frac{u}{\bar{u}}-1\right) d x+\int_{\mathbb{R}} \frac{\bar{\theta}}{\bar{u}} \partial_{t} u d y
\end{aligned}
$$

notice that

$$
\begin{aligned}
\int_{\mathbb{R}} \frac{\bar{\theta}}{\bar{u}} \partial_{t} u d y & =\int_{\mathbb{R}} \frac{\bar{\theta}}{\bar{u}} v_{y} d y \\
& =-\int_{\mathbb{R}}\left(\frac{\bar{\theta}}{\bar{u}}\right)_{y}(v-\bar{v}) d y+\int_{\mathbb{R}} \frac{\bar{\theta}}{\bar{u}} \bar{v}_{y} d y .
\end{aligned}
$$

(2.32) together with (2.33) yields that

$$
I_{2} \leq-\frac{d}{d t} \int_{\mathbb{R}} \bar{\theta}\left(\frac{u}{\bar{u}}-\ln \frac{u}{\bar{u}}-1\right) d x+C .
$$

Summing up (2.28), (2.31) with (2.34), we get

$$
\left|\int_{0}^{T} \int_{\mathbb{R}} \frac{\theta}{u} v_{y} d y d t\right| \leq 2 \delta \int_{0}^{T} \int_{\mathbb{R}} \frac{v_{y}^{2}}{u} d y d t+C(\delta) \int_{0}^{T} \max _{x} \theta d t+C T,
$$

notice that $\bar{v}_{y}>0$. Thus by summing up Lemma 2.1 , Lemma $2.2,(2.27)$, and (2.35), we get

$$
\int_{0}^{T} \int_{\mathbb{R}} v_{y}^{2} d y d t \leq C(T)
$$


And from (2.10), the Sobolev imbedding theorem, and (2.36), it follows that

$$
\begin{aligned}
\int_{0}^{T}\left\{\int_{-M}^{M} v^{4} d y+\sup _{[-M, M]} v^{2}(\cdot, t)\right\} d t & \leq C(M) \int_{0}^{T} \sup _{[-M, M]} v^{2} d t \\
& \leq C(M) \int_{0}^{T} \int_{-M}^{M}\left(v^{2}+v_{y}^{2}\right) d y d t \\
& \leq C(M) .
\end{aligned}
$$

For $M \in \mathbb{N}$, let $\phi \in C_{0}^{\infty}(\mathbb{R})$ with $\phi(y)=1$ for $|y| \leq M-1 / 2$ and $\phi(y)=0$ for $|y| \geq M$. Multiplying Eq. (2.6) by $\phi$, we get

$$
c_{V}[\phi \theta]_{t}=\kappa\left[\frac{(\phi \theta)_{y}}{u}-\frac{\theta \phi_{y}}{u}\right]_{y}-\kappa \frac{\theta_{y} \phi_{y}}{u}+\sigma v_{y} \phi .
$$

Hence, we can decompose $\phi \theta$ into $\phi \theta=\Theta+\widetilde{\Theta}$ where $\Theta$ and $\widetilde{\Theta}$ satisfy the linear parabolic equations in the domain $(-M, M)$ :

$$
\begin{aligned}
& c_{V} \Theta_{t}=\kappa\left[\frac{\Theta_{y}}{u}\right]_{y}-\kappa \frac{\theta_{y} \phi_{y}}{u}+\sigma v_{y} \phi \\
& \Theta=0 \text { at } x= \pm M \\
& \left.\Theta\right|_{t=0}=\phi \theta_{0}
\end{aligned}
$$

and

$$
\begin{aligned}
& c_{V} \widetilde{\Theta}_{t}=\kappa\left[\frac{\widetilde{\Theta}_{y}}{u}\right]_{y}-\kappa\left[\frac{\theta \phi_{y}}{u}\right]_{y}, \\
& \widetilde{\Theta}=0 \text { at } x= \pm M, \\
& \left.\widetilde{\Theta}\right|_{t=0}=0 .
\end{aligned}
$$

Notice that $[1 / u]_{t}=-v_{y} / u^{2} \in L_{\text {loc }}^{2}\left(\mathbb{R}_{+} \times \mathbb{R}\right)$, and $\theta_{y} \phi_{y}, \sigma v_{y} \phi \in L_{\text {loc }}^{1}\left(\mathbb{R}_{+} \times \mathbb{R}\right)$ by $(2.36)$ and (2.26). Therefore, we can apply Theorem 2.5 in [21, the estimate (2.26)] to obtain

$$
\begin{aligned}
\left\|\Theta_{y}\right\|_{L^{p}((0, T) \times(-M, M))} & \leq C(M, p)\left(\left\|\theta_{0}\right\|_{L^{1}(-M, M)}+\left\|\left(\theta_{y} \phi_{y}, \sigma v_{y} \phi\right)\right\|_{L^{1}((0, T) \times(-M, M))}\right. \\
& \leq C(M), \quad \text { for all } p<3 / 2 .
\end{aligned}
$$

To bound $\widetilde{\Theta}$, we multiply $(2.39)$ by $\widetilde{\Theta}$ in $L^{2}((-M, M) \times(0, T))$, integrate by parts, and employ (2.25) to arrive at

$$
\int_{0}^{T} \int_{-M}^{M} \widetilde{\Theta}_{y}^{2} d y d t \leq C \int_{0}^{T} \int_{-M}^{M} \theta^{2} d y d t \leq C(M),
$$

which, combined with $(2.40)$, results in $(2.24)_{3}$. This completes the proof.

3. Proof of Theorem 1.1. In this section, using the a priori estimates established in Sec. 1 and the method of weak convergence, we pass to the limit and consequently prove the existence of global weak solutions to (1.1)-(1.4). 
Let $\left(\rho^{\epsilon}(x, t), v^{\epsilon}(x, t), \theta^{\epsilon}(x, t)\right)$ denote the approximate solutions of (1.1)-(1.4) given at the beginning of Sec. 1 by mollifying the initial data. First we observe that in Eulerian coordinates $(x, t)$, Lemma 2.2 implies

$$
C_{1} \leq \rho^{\epsilon}(x, t) \leq C_{2}, C_{1} \leq \theta^{\epsilon}(x, t) \quad \forall x \in \mathbb{R}, t \in[0, T]
$$

and (3.1), (2.16), Lemmas 2.3 and 2.1 imply that for any $M \in \mathbb{N}$,

$$
\begin{aligned}
& \int_{0}^{T} \sup _{\mathbb{R}} \theta^{\epsilon}(\cdot, t) d t \leq C, \int_{0}^{T} \int_{\mathbb{R}}\left|v_{x}^{\epsilon}\right|^{2} d x d t \leq C(T), \\
& \int_{0}^{T} \int_{-M}^{M}\left\{\left|v^{\epsilon}\right|^{4}+\left|\theta^{\epsilon}\right|^{2}\right\} d x d t \leq C(M), \\
& \int_{0}^{T} \int_{-M}^{M}\left|\theta_{x}^{\epsilon}\right|^{p} d x d t \leq C(M, p), \quad p<3 / 2,
\end{aligned}
$$

and

$$
\begin{gathered}
\int_{\mathbb{R}}\left\{\left(\frac{\tilde{\rho}(x, t)}{\rho^{\epsilon}(x, t)}-\log \frac{\tilde{\rho}(x, t)}{\rho^{\epsilon}(x, t)}-1\right)+\left(\frac{\theta^{\epsilon}(x, t)}{\bar{\theta}}-\log \frac{\theta^{\epsilon}(x, t)}{\bar{\theta}}-1\right)\right. \\
\left.+\left(v^{\epsilon}(x, t)-\tilde{v}(x, t)\right)^{2}\right\} d x \leq C \quad \forall t \in[0, T]
\end{gathered}
$$

where

$$
\tilde{\rho}(x, t):=\frac{1}{\bar{u}(r(x, t))} \equiv \frac{1}{\bar{u}(y)}, \tilde{v}(x, t):=\frac{1}{\bar{v}(r(x, t))} \equiv \frac{1}{\bar{v}(y)},
$$

and $r(x, t)$ is defined by $(2.3)$ with $\rho$ replaced by $\rho^{\epsilon}$.

Recalling the definition of $r(x, t)$ and $(\bar{\rho}(x), \bar{v}(x))$ in the introduction, using (3.1), one finds that $(\tilde{\rho}(x, t), \tilde{v}(x, t))=(\bar{\rho}(x), \bar{v}(x))=\left(\rho^{ \pm}, v^{ \pm}\right)$for all $\pm x \geq K$ and $t \in[0, T]$ uniformly in $\epsilon$, where $K$ is some large constant. Hence, from (3.3) and (3.1), it follows that

$$
\begin{gathered}
\int_{\mathbb{R}}\left\{\left(\frac{\bar{\rho}(x)}{\rho^{\epsilon}(x, t)}-\log \frac{\bar{\rho}(x)}{\rho^{\epsilon}(x, t)}-1\right)+\left(\frac{\theta^{\epsilon}(x, t)}{\bar{\theta}}-\log \frac{\theta^{\epsilon}(x, t)}{\bar{\theta}}-1\right)\right. \\
\left.+\left(v^{\epsilon}(x, t)-\bar{v}(x)\right)^{2}\right\} d x \leq C \quad \forall t \in[0, T] .
\end{gathered}
$$

By (3.1) and (3.2) we can extract a subsequence of $\left(\rho^{\epsilon}, v^{\epsilon}, \theta^{\epsilon}\right)$, still denoted by $\left(\rho^{\epsilon}, v^{\epsilon}, \theta^{\epsilon}\right)$, such that as $\epsilon \downarrow 0$, the following weak or weak-* convergence holds:

$$
\begin{aligned}
& \rho^{\epsilon} \rightarrow \rho \text { in } L_{\mathrm{loc}}^{\infty}\left(\mathbb{R}_{+}, L^{\infty}\right), \theta^{\epsilon} \rightarrow \theta \text { in } L_{\mathrm{loc}}^{2}\left(\mathbb{R}_{+} \times \mathbb{R}\right) \cap L_{\mathrm{loc}}^{p}\left(\mathbb{R}_{+}, W_{\mathrm{loc}}^{1, p}\right)(p<3 / 2), \\
& v^{\epsilon} \rightarrow v \text { in } L_{\mathrm{loc}}^{\infty}\left(\mathbb{R}_{+}, L_{\mathrm{loc}}^{2}\right) \cap L_{\mathrm{loc}}^{2}\left(\mathbb{R}_{+}, H_{\mathrm{loc}}^{1}\right) \cap L_{\mathrm{loc}}^{4}\left(\mathbb{R}_{+} \times \mathbb{R}\right),
\end{aligned}
$$

and

$$
\begin{aligned}
& \rho^{\epsilon} v^{\epsilon} \rightarrow \overline{\rho v} \text { in } L_{\mathrm{loc}}^{\infty}\left(\mathbb{R}_{+}, L_{\mathrm{loc}}^{2}\right), \quad \rho^{\epsilon}\left(v^{\epsilon}\right)^{2} \rightarrow \overline{\rho v^{2}} \text { in } L_{\mathrm{loc}}^{2}\left(\mathbb{R}_{+} \times \mathbb{R}\right), \\
& \rho^{\epsilon} \theta^{\epsilon} \rightarrow \overline{\rho \theta} \text { in } L_{\mathrm{loc}}^{2}\left(\mathbb{R}_{+} \times \mathbb{R}\right), \quad \rho^{\epsilon} v^{\epsilon} \theta^{\epsilon} \rightarrow \overline{\rho v \theta} \text { in } L_{\mathrm{loc}}^{4 / 3}\left(\mathbb{R}_{+} \times \mathbb{R}\right), \\
& \rho^{\epsilon}\left(v^{\epsilon}\right)^{3} \rightarrow \overline{\rho v^{3}} \text { in } L_{\mathrm{loc}}^{4 / 3}\left(\mathbb{R}_{+} \times \mathbb{R}\right),
\end{aligned}
$$

where $\overline{\rho v}, \overline{\rho v^{2}}, \overline{\rho \theta}, \overline{\rho v \theta}$, and $\overline{\rho v^{3}}$ denote the weak or weak-* limit of $\rho^{\epsilon} v^{\epsilon}, \rho^{\epsilon}\left(v^{\epsilon}\right)^{2}, \rho^{\epsilon} \theta^{\epsilon}, \rho^{\epsilon} v^{\epsilon} \theta^{\epsilon}$, and $\rho^{\epsilon}\left(v^{\epsilon}\right)^{3}$, respectively. 
By the convexity of the function $x-\log x-1$ and (3.4), the inequality (3.4) still holds for the limit functions; that is

$$
\int_{\mathbb{R}}\left\{\left(\frac{\bar{\rho}}{\rho}-\log \frac{\bar{\rho}}{\rho}-1\right)+\left(\frac{\theta}{\bar{\theta}}-\log \frac{\theta}{\bar{\theta}}-1\right)+(v-\bar{v})^{2}\right\}(x, t) d x \leq C
$$

for $t \in[0, T]$, which, combined with (3.1) and Taylor's expansion, gives

$$
\int_{\mathbb{R}}(\rho-\bar{\rho})^{2}(x, t) d x \leq C \quad \forall t \in[0, T] .
$$

Now in order to justify that $(\rho, v, \theta)$ is indeed a weak solution of (1.1)-(1.4), in view of the definition of the weak solutions we have to show that

$$
\begin{gathered}
\overline{\rho v}=\rho v, \quad \overline{\rho v^{2}}=\rho v^{2}, \quad \overline{\rho \theta}=\rho \theta, \quad \overline{\rho v \theta}=\rho v \theta, \quad \overline{\rho v^{3}}=\rho v^{3}, \\
\left(v^{\epsilon}\right)^{2} \rightarrow v^{2} \text { strongly in } L_{\text {loc }}^{2}\left(\mathbb{R}_{+} \times \mathbb{R}\right)
\end{gathered}
$$

hold. In the following we apply Lemma 5.1 in $[15$, p. 12] to justify (3.8).

First, by Eqs. (1.1), (3.1), and (3.4), we find that

$$
\partial_{t} \rho^{\epsilon}=\partial_{x}\left(\rho^{\epsilon} v^{\epsilon}\right) \text { uniformly bounded in } L^{\infty}\left(\mathbb{R}_{+}, H_{\mathrm{loc}}^{-1}\right),
$$

and by $(3.2)_{3}$, we have

$$
\left\|v^{\epsilon}-v^{\epsilon}(\cdot+\xi, t)\right\|_{L^{2}\left([0, T], L_{\mathrm{loc}}^{\infty}\right)} \rightarrow 0 \text { as }|\xi| \rightarrow 0, \text { uniformly in } \epsilon .
$$

Thus by (3.5), (3.9), (3.10), and Lemma 5.1 in [15], we conclude that $\rho^{\epsilon} v^{\epsilon} \rightarrow \rho v$ in the sense of distributions, and therefore $\overline{\rho v}=\rho v$. Since by virtue of (3.1), (3.2), and (3.4), $\left\{\rho^{\epsilon} v^{\epsilon}\right\}$ is uniformly bounded in $L_{\text {loc }}^{\infty}\left(\mathbb{R}_{+}, L_{\text {loc }}^{2}\right)$ and

$$
\partial_{t}\left(\rho^{\epsilon} v^{\epsilon}\right)=-\partial_{x}\left[\rho^{\epsilon}\left(v^{\epsilon}\right)^{2}\right]-R \partial_{x}\left(\rho^{\epsilon} \theta^{\epsilon}\right)+\mu \partial_{x}^{2} v^{\epsilon}
$$

is uniformly bounded in $L_{\text {loc }}^{2}\left(\mathbb{R}_{+}, H_{\text {loc }}^{-1}\right)$, we obtain in the same manner that $\overline{\rho v^{2}}=\rho v^{2}$.

By virtue of $(3.2)_{3}$ and the mean value theorem, $\left\|\theta^{\epsilon}-\theta^{\epsilon}(\cdot+\xi, t)\right\|_{L^{p}\left([0, T], L_{\text {loc }}^{\infty}\right)} \rightarrow 0$ $(p<3 / 2)$ as $|\xi| \rightarrow 0$, uniformly in $\epsilon$. Thus again applying (3.5), (3.11), and Lemma 5.1 of [15], one obtains $\rho^{\epsilon} v^{\epsilon} \theta^{\epsilon} \rightarrow \rho v \theta$ in the sense of distributions. Hence $\overline{\rho v \theta}=\rho v \theta$. In the same way we can show that $\overline{\rho \theta}=\rho \theta$.

By (3.1), (3.2), and Eq. (1.3), one sees that $\partial_{t}\left\{\rho^{\epsilon}\left(\frac{\left(v^{\epsilon}\right)^{2}}{2}+c_{V} \theta^{\epsilon}\right)\right\}$ is uniformly bounded in

$$
L_{\text {loc }}^{4 / 3}\left(\mathbb{R}_{+}, W_{\mathrm{loc}}^{-1,4 / 3}\right)+L_{\mathrm{loc}}^{2}\left(\mathbb{R}_{+}, H_{\mathrm{loc}}^{-2}\right),
$$

and $\rho^{\epsilon}\left(\frac{\left(v^{\epsilon}\right)^{2}}{2}+c_{V} \theta^{\epsilon}\right)$ is uniformly bounded in $L_{\text {loc }}^{2}\left(\mathbb{R}_{+} \times \mathbb{R}\right)$. Thus by (3.10) and Lemma 5.1 in $[15]$,

$$
\rho^{\epsilon}\left(\frac{\left(v^{\epsilon}\right)^{2}}{2}+c_{V} \theta^{\epsilon}\right) \cdot v^{\epsilon}-\overline{\rho\left(\frac{v^{2}}{2}+c_{V} \theta\right)} \cdot v=\rho\left(\frac{v^{2}}{2}+\theta\right) \cdot v,
$$

which implies $\overline{\rho v^{3}}=\rho v^{3}$, where we have used the fact $\rho^{\epsilon} v^{\epsilon} \theta^{\epsilon} \rightarrow \rho v \theta$, and $\overline{\rho\left(\frac{v^{2}}{2}+c_{V} \theta\right)}$ denotes the weak limit of $\rho^{\epsilon}\left(\frac{\left(v^{\epsilon}\right)^{2}}{2}+\theta^{\epsilon}\right)$.

Finally, we show $\left(v^{\epsilon}\right)^{2} \rightarrow v^{2}$. By (3.10) and (3.4), one sees that

$$
\begin{aligned}
& \left\|\left(v^{\epsilon}\right)^{2}-\left(v^{\epsilon}\right)^{2}(\cdot+\xi, t)\right\|_{L^{2}\left([0, T], L_{\mathrm{loc}}^{2}\right)} \\
& \quad \leq 2\left\|v^{\epsilon}-v^{\epsilon}(\cdot+\xi, t)\right\|_{L^{2}\left([0, T], L_{\mathrm{loc}}^{\infty}\right)}\left\|v^{\epsilon}\right\|_{L^{\infty}\left([0, T], L_{\mathrm{loc}}^{2}\right)} \\
& \quad \leq C\left\|v^{\epsilon}-v^{\epsilon}(\cdot+\xi, t)\right\|_{L^{2}\left([0, T], L_{\mathrm{loc}}^{\infty}\right)} \rightarrow 0 \text { as }|\xi| \rightarrow 0, \text { uniformly in } \epsilon .
\end{aligned}
$$


From (3.12), (3.9), Lemma 5.1 in [15], and the fact that $\rho^{\epsilon}\left(v^{\epsilon}\right)^{2} \rightarrow \rho v^{2}$ and $\rho>0$, one gets

$$
\rho v^{2}<\rho^{\epsilon}\left(v^{\epsilon}\right)^{2} \rightarrow \rho \overline{v^{2}}
$$

which gives $\overline{v^{2}}=v^{2}$. Hence, by Theorem 1 of $[5], v^{\epsilon}(x, t) \rightarrow v(x, t)$ strongly in $L_{\text {loc }}^{2}\left(\mathbb{R}_{+} \times\right.$ $\mathbb{R})$. We have proved (3.8).

Now we multiply the system $(1.1)-(1.3)$ with $(\rho, v, \theta)$ replaced by $\left(\rho^{\epsilon}, v^{\epsilon}, \theta^{\epsilon}\right)$ by $\phi \in$ $C_{0}^{\infty}\left(\mathbb{R}_{+} \times \mathbb{R}\right)$, integrate over $(0, \infty) \times \mathbb{R}$, and integrate by parts. Letting $\epsilon \downarrow 0$, applying $(3.5),(3.6)$, and (3.8), and summing up the results for $(\rho, v, \theta)$ obtained above, we see that $(\rho, v, \theta)$ obtained in (3.5) is indeed a weak solution of (1.1)-(1.4). Finally, the estimate (1.10) follows from (3.1) and (3.7). This completes the proof of Theorem 1.1.

Acknowledgement. The authors would like to thank Professor A. A. Zlotnik for useful comments. This work was supported by the Special Funds for Major State Basic Research Projects (No. G1999032801), the NNSF, the CAEP, and the innovation grants from CAS.

\section{REFERENCES}

[1] S. N. Antontsev, A. V. Kazhikhov, and V. N. Monakhov, "Boundary Value Problems in Mechanics of Nonhomogeneous Fluids," North-Holland, Amsterdam, New York, 1990

[2] A. A. Amosov and A. A. Zlotnik, Global generalized solutions of the equations of the onedimensional motion of a viscous heat-conducting gas, Soviet Math. Dokl. 38, 1-5 (1989)

[3] A. A. Amosov and A. A. Zlotnik, Solvability "in the large" of a system of equations of the onedimensional motion of an inhomogeneous viscous heat-conducting gas, Math. Notes 52, 753-763 (1992)

[4] G. Q. Chen, D. Hoff, and K. Trivisa, Global solutions of the compressible Navier-Stokes equations with large discontinuous initial data, Comm. Partial Differential Equations 25, 2233-2257 (2000)

[5] L. C. Evans, "Weak Convergence Methods for Nonlinear Partial Differential Equations," CBMS No. 74, AMS, 1990

[6] H. Fujita-Yashima, M. Padula, and A. Novotný, Equation monodimensionnelle d'un gaz visqueux et calorifère avec des conditions initiales moins restrictives, Ricerche di Matematica XLII, 199-248 (1993)

[7] D. Hoff, Global well-posedness of the Cauchy problem for the Navier-Stokes equations of nonisentropic flow with discontinuous initial data, J. Diff. Eqs. 95, 33-74 (1992)

[8] D. Hoff, Global solutions of the equations of one-dimensional, compressible flow with large data and forces, and with differing end states, Z. Angew. Math. Phys. 49, 774-785 (1998)

[9] D. A. Iskenderova and Sh. S. Smagulov, The Cauchy problem for the equations of a viscous heatconducting gas with degenerate density, Comput. Math. Phys. 33, 1109-1117 (1993)

[10] S. Jiang, Global spherically symmetric solutions to the equations of a viscous polytropic ideal gas in an exterior domain, Comm. Math. Phys. 178, 339-374 (1996)

[11] S. Jiang, Large-time behavior of solutions to the equations of a one-dimensional viscous polytropic ideal gas in unbounded domains, Comm. Math. Phys. 200, 181-193 (1999)

[12] Y. I. Kanel, Cauchy problem for the equations of gas dynamics with viscosity, Siberian Math. J. 20, 208-218 (1979)

[13] A. V. Kazhikhov, Cauchy problem for viscous gas equations, Siberian Math. J. 23, $44-49$ (1982)

[14] A. V. Kazhikhov and V. V. Shelukhin, Unique global solution with respect to time of initial boundary value problems for one-dimensional equations of a viscous gas, J. Appl. Math. Mech. 41, 273-282 (1977)

[15] P. L. Lions, "Mathematical topics in Fluid Mechanics, Vol. 2, Compressible Models," Clarendon Press, Oxford, 1998

[16] T. Nagasawa, On the one-dimensional motion of the polytropic ideal gas non-fixed on the boundary, J. Diff. Eqs. 65, 49-67 (1986) 
[17] T. Nagasawa, On the asymptotic behavior of the one-dimensional motion of the polytropic ideal gas with stress-free condition, Quart. Appl. Math. 46, 665-679 (1988)

[18] T. Nagasawa, On the one-dimensional free boundary problem for the heat-conductive compressible viscous gas, In: Mimura, M., Nishida, T. (eds.), Recent Topics in Nonlinear PDE IV, Lecture Notes in Num. Appl. Anal. 10, pp. 83-99, Kinokuniya/North-Holland, Amsterdam, Tokyo, 1989

[19] M. H. Protter and H. F. Weinberger, "Maximum Principles in Differential Equations," Prentice Hall, Englewood Cliffs, New Jersey, 1967

[20] D. Serre, Sur l'équation monodimensionnelle d'un fluids visqueux, compressible et conducteur de chaleur, C. R. Acad. Sci. Paris, Sér. I 303, 703-706 (1986)

[21] A. A. Zlotnik and A. A. Amosov, On stability of generalized solutions to the equations of onedimensional motion of a viscous heat-conducting gas, Siberian Math. J. 38, 663-684 (1997) 